\title{
IEEE 802 Standardization on Heterogeneous Network Interworking
}

\author{
Hyunho Park****, Hyeong Ho Lee***, Seung-Hwan Lee* \\ *ETRI (Electronics and Telecommunications Research Institute), Korea \\ **Department of Broadband Network Technology, UST (University of Science and Technology), Korea \\ hyunhopark@etri.re.kr, holee@etri.re.kr, Ish@etri.re.kr
}

\begin{abstract}
As various radio access technologies and multi-mode terminals incorporating multiple network interfaces appear, research and development interest on heterogeneous network interworking increases. IEEE 802 groups have standardized technologies on interworking between heterogeneous IEEE 802 networks and between an IEEE 802 network and a cellular network. This paper analyzes technologies on heterogeneous network interworking of IEEE 802 standards and provides trends and requirements of future technology on heterogeneous network interworking.
\end{abstract}

Keywords—IEEE 802, Interworking, Heterogeneous network, MIH, WLAN

\section{INTRODUCTION}

Multi-mode terminals (MMTs) such as smart phones and tablet PCs, which have network interfaces of various radio access technologies, have become popular in recent days. Moreover, various wireless networks such as WLAN, WPAN, WiMAX, and cellular networks (WCDMA, HSDPA, HSPA, and LTE networks) have been deployed widely. Based on the network environments, users of MMTs want to connect to the wireless network that can meet the users' requirements such as communication cost, quality of experience (QoE), and power saving of the MMTs. Also, network operators want to distribute network load of a wireless network to other wireless networks to cope with explosion of data traffic. To meet the requirements of users and network operators, research and development interests on interworking among heterogeneous networks such as vertical handover and data offload have increased. Standard organizations such as IEEE 802, 3GPP, and IETF also have standardized technologies on heterogeneous network interworking.

This paper analyzes IEEE 802 standards on heterogeneous network interworking and explains standardization activities of IEEE 802 working groups relevant to the interworking functions. Firstly, we introduce standards and standardization activities of IEEE 802.21 working group (WG) and its task groups. IEEE 802.21 working group standardized Media Independent Handover (MIH) that provides seamless handover in heterogeneous networks, security extensions to MIH services and protocol, and MIH extension for supporting handover between communication and broadcast networks [1, 2, 3]. Moreover, it has been producing draft standards on optimized single radio handover and multicast group management based on MIH protocol. IEEE 802.21 WG launched new standardization projects on framework and services of MIH technology [4, 5]. The other IEEE 802 groups such as IEEE 802.11 high efficiency WLAN (HEW) study group (SG) and omni-range area networks (OmniRAN) executive committee study group (ECSG) also study network architecture and use cases for heterogeneous network interworking $[6,7]$.

Based on analysis of IEEE 802 standards, we will discuss future technical trends and requirements for IEEE 802 heterogeneous network interworking. The future interworking technologies will enable network assistance for device-todevice (D2D) communication, integration between IEEE 802 networks or between IEEE 802 network and cellular network, and resource management in the integrated heterogeneous networks. Requirements of future interworking technologies will include improvement of users' satisfaction, power saving of multi-mode terminals, and integration of IEEE 802 access networks. This paper will be a valuable reference for research and development of interworking service in IEEE 802 heterogeneous networks.

The remainders of the paper are organized as follows. In section II, we analyze standard works of IEEE 802.21 WG and introduce issues of heterogeneous network interworking discussed in the group. Section III explains current issues of other IEEE 802 groups such as IEEE 802.11 HEW SG and OmniRAN ECSG. In section IV, we offer concluding remarks based on the analysis of IEEE802 standards and standardization works in IEEE 802 groups.

\section{IEEE 802.21 STANDARDIZATION WORKS FOR HETEROGENEOUS NETWORK INTERWORKING}

IEEE 802.21 WG published a standard on media independent handover (MIH) technology, which provides seamless handover in heterogeneous networks. Afterwards, its task groups such as task groups a, b, c, and d have standardized technologies to solve additional issues on handover in heterogeneous networks. Task group $\mathrm{m}$ and 802.21.1 have discussed framework and services of MIH technology and various interworking issues in heterogeneous networks. This section explains standardization works in IEEE 802.21 WG, and forecasts future technologies and 
requirements for heterogeneous network interworking based on them.

\section{A. IEEE 802.21-2008 Standard}

IEEE 802.21-2008 standard, which was published in Jan. 2009, is a base document of other IEEE 802.21 standards and introduced MIH technology for the first time [1]. MIH technology can provide interaction between MIH user (network layer or higher layer than the network layer) and link layer (physical layer and data link layer), and thus reduce handover latency. The interaction provided by $\mathrm{MIH}$ technology is defined as MIH services. The MIH services include event service, command service, and information service. Event service and command service enable link layer to report its link status to $\mathrm{MIH}$ user and $\mathrm{MIH}$ user to control link layer, respectively. Information service is used to provide network configuration information to MMTs by using information servers that have network configuration information.

MIH function (MIHF) in Fig. 1 is a functional entity that realizes MIH services in mobile terminals and network entities. MIHF of a mobile terminal or a network entity can interact with MIH user, Link layer, and remote MIHF that does not belong to the same mobile terminal or same network entity. For these kinds of interactions, MIHF has interfaces called service access points (SAPs). MIH_SAP, MIH_LINK_SAP, and MIH_NET_SAP in Fig. 1 are SAPs to be used for interacting with $\mathrm{MIH}$ user, link layer, and remote MIHF. Interaction signals through MIH_SAP or MIH_LINK_SAP are defined as MIH primitives, and interaction signals through MIH_NET_SAP are defined as MIH messages.

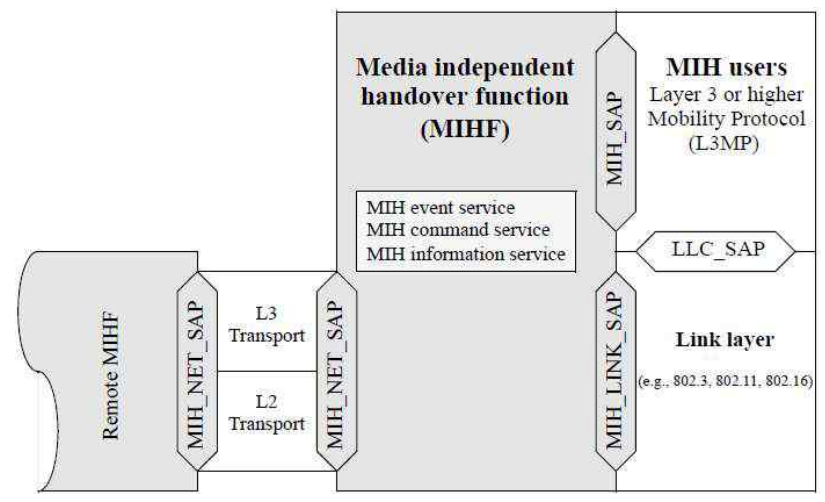

Figure 1. MIHF refernece model [1]

IEEE 802.21-2008 standard defines a mobile node (MN) as the mobile terminal (e.g., MMT) that can perform handover between heterogeneous networks, a point of attachment (PoA) as the network entity (e.g., base station in cellular networks and access point in WLAN) that establishes link layer connection with $\mathrm{MN}$, and an MIH point of service (PoS) as the network entity that supports MIHF. IEEE 802.21-2008 standard describes mobile-initiated handover triggered by an $\mathrm{MN}$ as well as network-initiated handover triggered by $\mathrm{MIH}$ PoS.

\section{B. IEEE 802.21a-2012 Standard}

IEEE 802.21 task group a (TGa) standardized security extension of MIH technology and then published it as IEEE 802.21a-2012 standard in May 2012 [2]. IEEE 802.21a-2012 standard provides $\mathrm{MIH}$ message protection and proactive authentication to extend security of MIH technology [8]. To protect MIH messages, IEEE 802.21a-2012 offers message protection by using security protocols (e.g., transport layer security (TLS) and datagram transport layer security (DTLS)) and authentication protocols (e.g., extensible authentication protocol (EAP) and EAP re-authentication protocol (ERP)). Proactive authentication is authentication with candidate network(s) prior to handover execution. The extended security of MIH in IEEE 802.21a-2012 standard can solve security problems and reduces duration for authentication, and thus reduce handover latency and packet loss when a mobile node performs handover in heterogeneous networks.

\section{IEEE 802.21b-2012 Standard}

IEEE 802.21 task group b (TGb) standardized handover technology between a communication network (e.g., WLAN, WiMAX network, and cellular network) and a broadcast network (e.g., digital video broadcasting (DVB) and digital multimedia broadcasting (DMB) networks), and published it as IEEE 802.21b-2012 standard in May 2012 [3]. IEEE $802.21 \mathrm{~b}-2012$ created a new message of command service, MIH_Net_HO_Bcst_Commit. It is used for network-initiated handover between communication and broadcast networks [8]. IEEE 802.21b-2012 standard enables a mobile node to perform seamless handover between communication and broadcast networks.

\section{IEEE 802.21c D7 Draft Standard}

IEEE 802.21 task group c (TGc) has been standardizing optimized single radio handover (SRHO) between heterogeneous networks from Jan. 2010, and produced IEEE 802.21c D7 draft standard recently [4]. SRHO means the handover executed by the MN that admits transmission over only one network interface at a time. The MN with SRHO consumes less power and results in less radio interference between network interfaces than the $\mathrm{MN}$ with dual radio handover that admits transmission over multiple network interfaces at a time $[9,10]$. Owing to the reduced radio interference, radio frequency (RF) filter of an $\mathrm{MN}$ can be designed simply and then cost of the MN can be reduced. However, while a network interface of the MN with simplified RF filter transfers data through a network, other network interfaces of the $\mathrm{MN}$ are difficult to listen to signals from other networks that the $\mathrm{MN}$ does not connect to, and thus the $\mathrm{MN}$ may not discover and connect to a target network. To resolve this problem, IEEE 802.21c draft standard provides optimized SRHO technology with proxy services that help the MN discover and connect to a target network.

Proxy services are composed of enhanced network discovery and preregistration. A proxy Information Server facilitates the enhanced network discovery by providing network configuration information to an $\mathrm{MN}$ that does not 
have authority for accessing to information server. Preregistration is registering an $\mathrm{MN}$ to a target network via a proxy target PoA (TPoA) before link layer handover execution.

Enhanced network discovery can be realized by a proxy Information Server that can access to both an $\mathrm{MN}$ and an information server. MN with SRHO may not search information server when the information server is located out of the source network that MN connects to. MN with SRHO also may not access to information server if the $\mathrm{MN}$ and information server use different network technologies or the information server does not allow signals from the MN. To provide network configuration information of the information server for the $\mathrm{MN}$ in these cases, the proxy Information Server receives the network configuration information by accessing to the information server instead of the $\mathrm{MN}$ and then forwards the information to the MN. Proxy Information Server can be achieved as source network PoS (SPoS) that is the MIH PoS located in source network.

$\mathrm{MN}$ interacts with proxy Information Server by use of MIH_CTRL_Transfer request and response messages. MIH_CTRL_Transfer request and response messages are $\mathrm{MIH}$ request and response messages encapsulating request and response messages for network configuration information, respectively. SRHO-capable PoA is the PoA that implements one or more command service messages between MIH_CTRL_Transfer and MIH_Prereg_Xfer messages [4, 11]. Figure 2 shows signaling procedure for enhanced network discovery as described below.

(a) $\mathrm{MN}$ requests network configuration information from proxy Information Server by using MIH_CTRL_Transfer request message or non-MIH request message. Non-MIH message is message used by other network configuration technology than MIH technology. Generic advertisement service (GAS) request message for access network query protocol (ANQP) can be an example of non-MIH message [12].

(b) SRHO-capable PoA delivers MIH_CTRL_Transfer request message to proxy Information Server. If the $\mathrm{MN}$ generates non-MIH request message in step (a), the SRHOcapable PoA transforms the non-MIH request message into MIH_CTRL_Transfer request message.

(c) The proxy Information Server performs signaling with information server. The step (c) is out of scope in IEEE $802.21 \mathrm{c}$ standard draft.

(d) The proxy Information Server encapsulates network configuration information from information server into MIH_CTRL_Transfer response message and then sends it to SRHO-capable PoA.

(e) If SRHO-capable PoA received MIH_CTRL_Transfer request message from $\mathrm{MN}$ in step (a), the SRHO-capable PoA forwards the MIH_CTRL_Transfer response message to the MN. Otherwise, the SRHO-capable PoA decapsulates the MIH_CTRL_Transfer response message, and sends the decapsulated non-MIH response message to the MN.
Preregistration means $\mathrm{MN}$ registers to target PoA (TPoA) that $\mathrm{MN}$ wants to connect with before link layer handover execution. TPoA means a PoA of target network that MN wants to connect to. Figure 3 shows procedure for preregistration. For registering $\mathrm{MN}$ to the TPoA, MN and TPoA interact with each other by use of link layer frames of target network. However, if MN with SRHO connects to source network, MN cannot transmit any link layer frame of target network interface over the air link and then also cannot request registration of the $\mathrm{MN}$ to TPoA. Alternatively, the $\mathrm{MN}$ can request registration to TPoA by sending MIH_Prereg_Xfer request message, which encapsulates link layer frame to proxy TPoA, through source network (step (a) in Fig. 3). The proxy TPoA registers MN to TPoA and can be realized as MIH PoS in target network. If TPoA implements MIH_Prereg_Xfer, proxy TPoA forwards MIH_Prereg_Xfer request message to TPoA, and then TPoA responds to the request message with $\mathrm{MIH}$ _Prereg_Xfer response message that encapsulates link layer frame of TPoA (step (b-1) in Fig. 3). Otherwise, other signaling than MIH_Prereg_Xfer is used for registering MN to TPoA (step (b-2) in Fig. 3). The step (b2) in Fig. 3 is out of scope in IEEE $802.21 \mathrm{c}$ draft standard. After step (b-1) or step (b-2) in Fig. 3, the proxy TPoA transmits MIH_Prereg_Xfer response message as response for MIH_Prereg_Xfer request message in step (a) of Fig. 3.

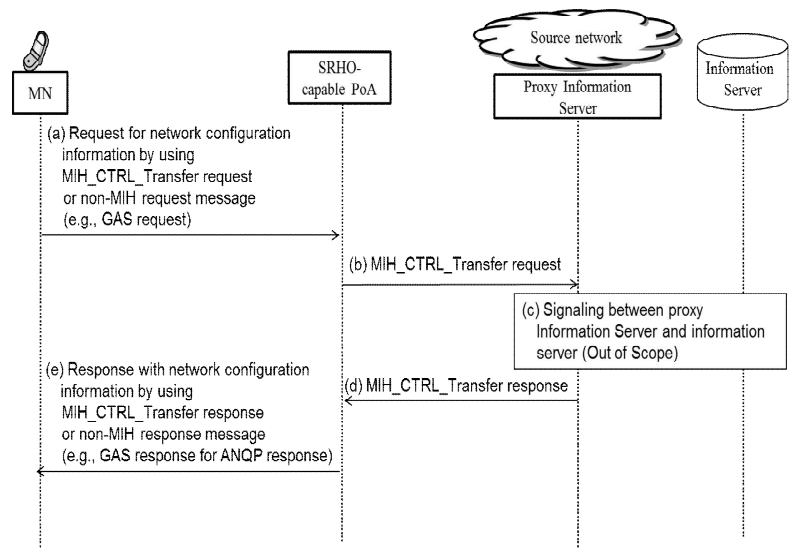

Figure 2. Enhanced network discovery with proxy Information Server [4]

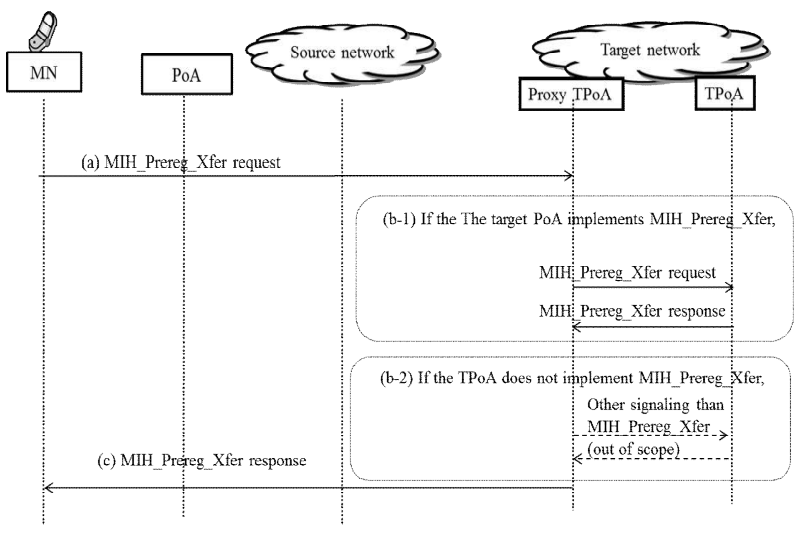

Figure 3. Preregistration with proxy TPoA [4] 
Proxy services of IEEE 802.21c draft standard are also valuable for future interworking technologies. For enhanced network discovery, proxy service provides interworking between MIH technology and other network configuration technologies such as ANQP. For preregistration, proxy service supports interworking between $\mathrm{MN}$ and target networks through source network. The extended interworking technologies of proxy services will be solutions to solve future interworking issues.

\section{E. IEEE 802.21d D2 Draft Standard}

IEEE 802.21 task group d (TGd) has been standardizing group handover for mesh networks (e.g., IEEE 802.15.4 network) and broadcast networks (e.g., DVB and DMB networks), and produced IEEE 802.21d D2 draft standard recently [5]. Group handover in IEEE 802.21d draft standard can solve communication failure (e.g., breakdown in base stations of cellular network), load balancing, and firmware update of group nodes.

\section{F. Future issues of heterogeneous network interworking in IEEE 802.21 task group $m$ and 802.21.1}

IEEE 802.21 task group $\mathrm{m}$ (TGm) and 802.21.1 (TG21.1) launched discussions on their own standard works from May 2013. Aim of TGm is producing IEEE $802.21 \mathrm{~m}$ standard that defines an extensible IEEE 802 media access independent services framework (i.e., function and protocol) that enables the optimization of handover and other services (e.g., discovery) between heterogeneous IEEE 802 networks and enables those between IEEE 802 networks and cellular networks. TGm has listed table of contents for IEEE $802.21 \mathrm{~m}$ standard and also discussed on some editorial errors to be modified in the existing standards [13]. Aim of TG21.1 is producing IEEE 802.21.1 standard that improves the user experience of mobile devices by describing the necessary media independent services to facilitate interworking between IEEE 802 networks and facilitate interworking between IEEE 802 networks and non IEEE 802 networks (e.g., cellular networks). The media independent services of IEEE 802.21.1 will include media independent services of the existing standards (IEEE 802.21-2008, IEEE 802.21a-2012, and IEEE 802.21b-2012 standards) and draft standards (IEEE 802.21c and IEEE 802.21d standards) as well as new media independent services for future interworking issues in heterogeneous networks.

TG21.1 has been studying some work items on heterogeneous network interworking. The first work item is network-assisted device-to-device (D2D) communication (NADC) based on architecture of IEEE 802.21-2008 standard [14]. The NADC can be accomplished by enhanced MIH PoS and $\mathrm{MIH}$ information server in heterogeneous networks as shown in Fig. 4. MN with the NADC can easily find its peer $\mathrm{MN}$ and connect to its peer MN. The NADC will offer network assistance for various D2D communications such as proximity service (ProSe) communication of 3GPP, Wi-Fi Direct, and IEEE 802.15.8 peer aware communication (PAC). TG21.1 approved the NADC as a work item for IEEE 802.21.1 standard in Nov. 2013.
The second work item is interworking between access networks [15]. As shown in Fig. 5, if an access network (e.g., Access Network A) are disconnected from core network due to some failures of the gateway or link (step (a)), the access network connects to other access network (e.g., Access Network B) and thus communicates with core network (step (b)). The interworking operation between access networks is similar to interworking between source and target networks with proxy service in IEEE 802.21c draft standard [4]. TG21.1 approved interworking between access networks as a work item for IEEE 802.21.1 standard in Nov. 2013.

The other topics than the two work items were not approved as work items by TG21.1 yet, but the other topics discussed in TG21.1 help us expect future issues on heterogeneous network interworking. TG21.1 has discussed $\mathrm{MIH} \mathrm{PoS}$ as software-defined networking (SDN) controller from Sep. 2013 [16]. In Nov. 2013, TG21.1 also discussed dynamic frequency channel allocation of WLAN to improve users' QoE [17]. These two topics imply that an important future issue on heterogeneous network interworking is resource management of heterogeneous networks for improving users' satisfaction. In Section III, we will show that some IEEE 802 groups other than IEEE 802.21 WG also pay attention to resource management for users' satisfaction as issues of heterogeneous network interworking.

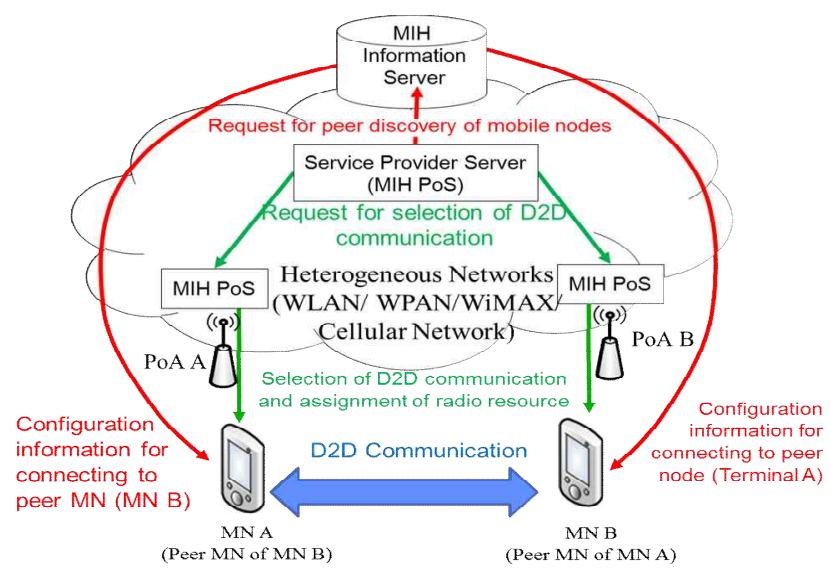

Figure 4. NADC based on MIH frame work [14]

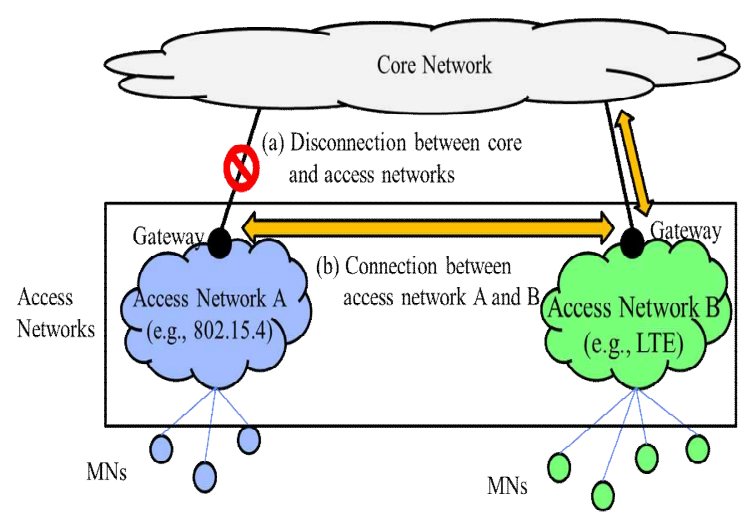

Figure 5. Interworking between access networks [15] 


\section{STANDARD WORKS OF OTHER IEEE 802 WGs}

Heterogeneous network interworking has been also discussed by some IEEE 802 groups other than IEEE 802.21 WG. IEEE 802.11 high efficiency WLAN (HEW) study group (SG) is discussing interworking between WLAN and cellular network, and omni-range area networks (OmniRAN) executive committee study group (ECSG) is discussing integration architecture of IEEE 802 networks. In this section, we will review activities of these groups and show that these two groups are studying resource management for users' satisfaction in heterogeneous networks.

\section{A. IEEE 802.11 HEW SG}

IEEE 802.11 HEW SG was established to study methods to improve spectrum efficiency and area throughput of WLAN in May 2013. IEEE 802.11 HEW SG produced a document of usage models in July 2013, and cellular traffic offloading to WLAN is one of the important usage models from radio resource management perspective [7]. IEEE 802.11 HEW SG has been discussing requirements for cellular traffic offload in public areas (e.g., airports and train stations) as described in Fig. 6 and private areas (e.g., office and lecture hall) [7, 18]. In addition, in order to increase area throughput, IEEE 802.11 HEW SG is also discussing D2D communication assisted by infrastructure WLAN $[19,20]$. Studies of IEEE 802.11 HEW SG imply that integrated resource management among cellular network, infrastructure WLAN, and D2D communication is necessary to improve satisfaction of users.

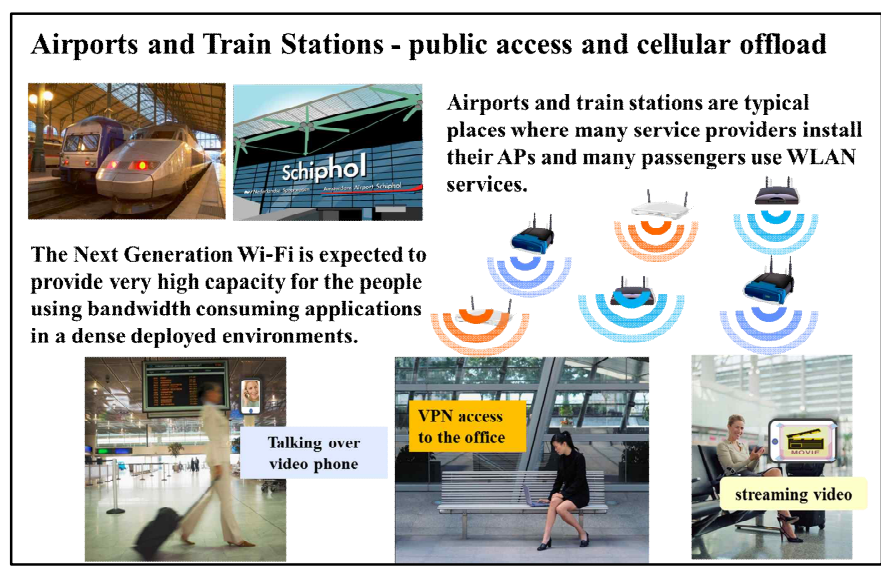

Figure 6. Cellular offload usage model in public area [7]

\section{B. OmniRAN ECSG}

IEEE 802.16 heterogeneous networks (Hetnet) SG launched to study on open mobile network interfaces from Mar. 2013, and then Omni-range area networks (OmniRAN) executive committee study group (ECSG) took over the study from IEEE 802.16 Hetnet $\mathrm{SG}$ in Nov. 2012. Aim of OmniRAN is producing standard for recommended practice that specifies an access network by utilizing technologies based on the family of IEEE 802 standards. Figure 7 describes the open mobile network interfaces (R1, R2, R3, R4, and R5) which are interfaces among core network, access network (AN), and mobile station (MS) that is equal to mobile terminal or mobile node [6]. OmniRAN has discussed applications of the open mobile network interfaces to S2a mobility based on GTP (SaMOG), software-defined networking (SDN), roaming in WLANs, and D2D communication [21, 22, 23]. These applications indicate that resource management of integrated heterogeneous networks will be required for improving satisfaction of users.

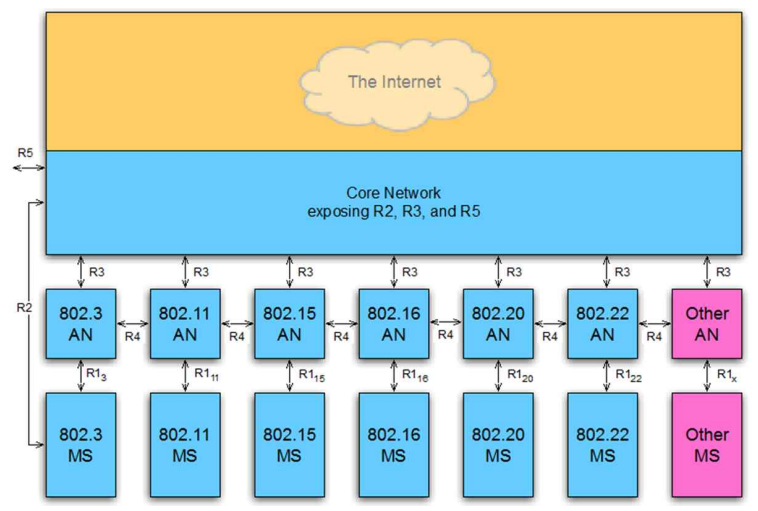

Figure 7. Open mobile network interfaces of OmniRAN ECSG [6]

\section{IV.CONCLUSIONS}

Development of multi-mode terminals and various network technologies leads to the necessity and requirements for heterogeneous network interworking from users' and network operators' perspectives. In heterogeneous networks, users require communications through networks that improve users' satisfaction, and network operators require efficient allocation of resources in heterogeneous networks. In order to meet users' and network operators' requirements, IEEE 802 groups have been developing interworking technologies of heterogeneous networks. IEEE 802.21 WG, which standardized handover between heterogeneous networks, developed technology standards that enhances security of the handover and enables communication and broadcast networks. IEEE 802.21 WG has also developed technology standards that enable fast handover with low power consumption and group handover. In recent days, the IEEE 802.21 WG has been studying various use cases for heterogeneous network interworking such as network-assisted D2D communication, interworking between access networks, dynamic frequency channel allocation, and resource management in softwaredefined networking. IEEE 802.11 HEW SG and OmniRAN ECSG are also studying resource management of heterogeneous networks.

Based on these technology trends, we can expect that the future technology of heterogeneous network interworking will be integrated mobility and resource managements of heterogeneous networks for improving users' satisfaction. Preexisting interworking technology of heterogeneous networks supports mobility and continuity of communication service for a mobile terminal, as in MIH technology of IEEE 802.21-2008. 
Recently, IEEE 802 groups are studying interworking technologies that will support resource management of heterogeneous networks. Therefore, we expect that integrated mobility and resource managements of heterogeneous networks will be required for future heterogeneous networks.

\section{ACKNOWLEDGMENT}

This research was funded by the MSIP (Ministry of Science, ICT \& Future Planning), Korea in the ICT R\&D Program 2013 ("Research on wireless transmission techniques for enhancing QoE performance of smart mobile services," 2013).

\section{REFERENCES}

[1] IEEE Standard for Local and metropolitan area networks- Part 21 Media Independent Handover Services, IEEE Std. 802.21 ${ }^{\mathrm{TM}}-2008$, Jan. 2009.

[2] IEEE Standard for Local and metropolitan area networks- Part 21: Media Independent Handover Services Amendment 1: Security Extensions to Media Independent Handover Services and Protocol, IEEE Std. 802.21 $\mathrm{a}^{\mathrm{TM}}-2012$, May 2012

[3] IEEE Standard for Local and metropolitan area networks- Part 21 Media Independent Handover Services Amendment 2: Extension for Supporting Handovers with Downlink Only Technologies, IEEE Std. $802.21 b^{\mathrm{TM}}-2012$, May 2012.

[4] Draft Standard for Local Metropolitan Area Networks-Part 21 Media Independent Handover Services Amendment 3: Optimized Single Radio Handovers, IEEE P802.21c/D7, Dec. 2013

[5] Draft Standard for Local Metropolitan Area Networks- Part 21 . Media Independent Handover Services Amendment 4: Multicast Group Management, IEEE P802.21d/D2, Sept. 2013.

[6] M. Riegel, "OmniRAN Overview and Status," IEEE 802-omniran12/0002r1, Nov. 2013

[7] L. Cariou, "Usage models for IEEE 802.11 High Efficiency WLAN study group (HEW SG) - Liaison with WFA," IEEE 802.11-13/0657r6, Jul. 2013

[8] B. S. Ghahfarokhi and N. Movahhedinia, "A survey on applications of IEEE 802.21 Media Independent Handover framework in next generation wireless networks," Computer Communications, vol. 36, pp. 1101-1119, Jun. 2013.

[9] H. Park and H. H. Lee, "Smart WLAN Discovery for Power Saving of Dual-Mode Terminals," ETRI Journal, vol. 35, no. 6, pp.1144-1147, Dec. 2013.

[10] 3rd Generation Partnership Project; Technical Specification Group Radio Access Network; Evolved Universal Terrestrial Radio Access (E UTRA); Study on signalling and procedure for interference avoidance for in-device coexistence (Release 11), 3GPP TR 36.816 V11.2.0, Dec. 2011.

[11] H. Park, H. H. Lee, and H. A. Chan, "Gateway Service for Integration of Heterogeneous Networks using Different Interworking Solutions,' in Proc. ICACT'13, pp.489-494, Jan. 2013.

[12] IEEE Standard for Information technology-Telecommunications and information exchange between systems Local and metropolitan area networks-Specific requirements Part 11: Wireless LAN Medium Access Control (MAC) and Physical Layer (PHY) Specifications, IEEE Std. 802.11 $1^{\mathrm{TM}}-2012$, Mar. 2012.

[13] C. E. Perkins, "802.21-2008 text to be included," IEEE 802.2113/0210r0, Nov. 2013.

[14] H. Park, H. Lee, S. Lee, and J. Choi, "MIH Service Use Cases for Network-Assisted D2D Communication of Service Providers and Network Operators," IEEE 802.21-13/0196r0, Nov. 2013.

[15] Y. Ohba, R. Salazar, R. Turner, and S. Das, "Interworking Service Architecture and Requirements," IEEE 802.21-13/0174r0, Sep. 2013.

[16] C. E. Perkins, "ONF Wireless \& Mobility Use Case Proposal," IEEE 802.21-13/0173r0, Sep. 2013.

[17] H. Park, H. Lee, S. Lee, and J. Choi, "MIH Service Use Cases for Dynamic Frequency Channel Allocation of IEEE 802.11 WLANs," IEEE 802.21-13/0197r0, Nov. 2013.
[18] H. Tian, B. Liu, X. Zhu, B. Wang, H. Qin, B. Fan, and Z. Wang, "WLAN Cellular Offload in High Speed Moving Environment," IEEE 802.11-13/1169r0, Sep. 2013.

[19] T. Hui, W. Zhibo, and C. Nannan, "Potential Solutions to D2D Assisted WLAN," IEEE 802.11-13/0631r0, May. 2013.

[20] Z. Wu, X. Gao, and T. Peng, "D2D Technology for HEW," IEEE 802.11-13/1089r1, Sep. 2013.

[21] H. Park and H. Lee, "OmniRAN Proximity Service Use Case," IEEE 802-omniran-13/0016r2, Mar. 2013.

[22] M. Riegel, "OmniRAN SaMOG Use Case," IEEE 802-omniran13/0017r1, Mar. 2013

[23] M. Riegel, "OmniRAN SDN Use Case for External Communication," IEEE 802-omniran-13/00060-r0, Aug. 2013.

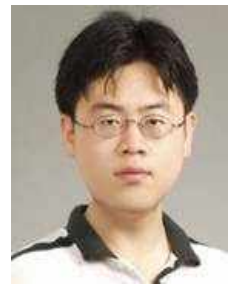

Hyunho Park received the B.S. degree in electrical engineering and computer science from Kyungpook National University, Daegu, Rep. of Korea, in 2005 and the M.S. degree in information and communications from the Gwangju Institute of Science and Technology, Gwangju, Rep. of Korea, in 2007. He is currently working towards his $\mathrm{PhD}$ degree in Broadband Network Technology at the University of Science and Technology, Rep. of Korea. From 2008 he has been working for ETRI (Electronics and Telecommunications Research Institute) and engaged in the research and standardization on vertica handover technologies for heterogeneous networks. He is serving as a secretary of IEEE Standard 802.21c Task Group on Single Radio Handover Optimization. His research interests include vertical handover and interworking between heterogeneous networks.

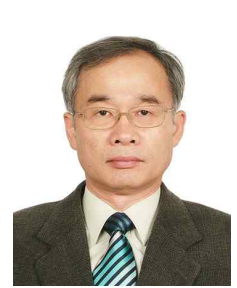

Hyeong Ho Lee received B.S. degree from Seoul National University, Seoul, Korea in 1977, and the M.S and Ph.D. degrees from KAIST (Korea Advanced Institute of Science and Technology), Daejeon, Korea, all in Electrical Engineering in 1979 and 1983, respectively. From 1983 he has been working for ETRI (Electronics and Telecommunications Research Institute) and engaged in the research and development of digital switching systems, LAN equipment, routers, optical access systems, and IT standardization. From 1984 to 1986, he was a visiting engineer in AT\&T Bell Laboratories, Naperville, U.S.A., where he was involved in the development of the No.5 ESS digital switching system. From 1997 to 2007, he was the director of Switching System, Router Technology, Optical Access Network Technology Departments, and Protocol Engineering Center (PEC) in ETRI. Since 2008, he is serving as a Special Fellow in ETRI, and works in the area of standardization researches for the next generation IT environment. Since 2013, he has been an adjunct professor at UST (University of Science and Technology), Daejeon, Korea. He was the chairman of KOREF (Korea Ethernet Forum) from 2000 to 2004, the President of IPv6 Forum Korea from 2005 to 2008, and a Vice President of IEEK (Institute of Electronics Engineers of Korea) from 2004 to 2011. Also, from 2005 to 2012, He served as a Vice Chairman of ITU-T SG11 (Study Group on signalling requirements, protocols and test specifications). Currently, he is the Chairman of IEEE Korea Council, an Editor of the ETRI Journal, a Council member of IEEK, a Council member of KICS (Korea Information and Communications Society), and a Senior member of IEEE (Institute of Electrical and Electronics Engineers).

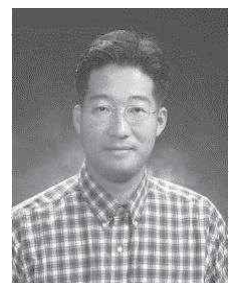

Seung-Hwan Lee received the BS and MS degrees from Korea University, Seoul, Korea, in 1995 and 1997, respectively, and the $\mathrm{PhD}$ degree from the University of Edinburgh, Edinburgh, UK in 2007. He has been with the Electronics and Telecommunications Research Institute (ETRI) since 2001. He has been an adjunct professor at the University of Science and Technology since 2010 . He is now a head of the integrated wireless research section at ETRI and in charge of developing integrated wireless technologies for the $5 \mathrm{G}$ system. 